\title{
Optimisation of solid liquid extraction of jatropha oil using petroleum ether.
}

\begin{abstract}
Jatropha curcas I. is an oil-bearing seed plant with a wide range of applications. The oil from the seeds of this plant has been used as an industrial raw material for many years. One of the important characteristics of jatropha oil is its potential for fuel and biodiesel production to meet the global energy demand. In this paper, solid-liquid extraction of jatropha oil from seeds using petroleum ether was optimised on the basis of the amount of the extracted oil. Four main factors, namely temperature, the solvent-to-solid ratio, the reaction time and the size of the raw material, were investigated to optimise extraction conditions for achieving the highest oil yield under experimental conditions. The kinetics of the extraction using petroleum ether as the solvent were also studied and fitted to a second-order model. The free fatty acid (FFA) content of the oil was used as an index of the oil quality. The optimum conditions were found to be $7 \mathrm{~h}$ of reaction time, a temperature of $68^{\circ} \mathrm{C}$, a coarse particle size of $0.5-0.75 \mathrm{~mm}$ and a solvent-to-solid ratio of $6: 1$. Storing the ground seeds for one week before extraction had a remarkably negative effect on the quality of the oil produced (FFA $\approx 6.99 \%$ ), whereas the quality of the oil was satisfactory when extracted from the seeds immediately after grinding (FFA $\approx 0.62 \%$ ). The experimental data fitted well to the secondorder model with the saturation extraction capacity and the initial extraction rate increasing with increasing temperature.
\end{abstract}

Keyword: Jatropha; Extraction; Petroleum ether; Biodiesel 